\title{
Analysis of Handover Frequencies for Predictive, Reactive and Proxy Schemes and Their Implications on IPv6 and Multicast Mobility
}

\author{
Thomas C. Schmidt ${ }^{1,2}$ and Matthias Wählisch ${ }^{2}$ \\ 1 HAW Hamburg, FB Elektrotechnik und Informatik, \\ Berliner Tor 7, 20099 Hamburg \\ 2 FHTW Berlin, Hochschulrechenzentrum, \\ Treskowallee 8, 10318 Berlin \\ \{schmidt, mw\}@fhtw-berlin.de
}

\begin{abstract}
Handovers in mobile packet networks commonly produce packet loss, delay and jitter, thereby significantly degrading network performance. Mobile IPv6 handover performance is strongly topology dependent and results in inferior service quality in wide area scenarios. To approach seamless mobility in IPv6 networks predicitive, reactive and proxy schemes have been proposed for improvement. In this article we analyse and compare handover frequencies for the corresponding protocols, as they are an immediate measure on performance quality. Using analytical methods as well as stochastic simulations of walking users within a cell geometry, we calculate the expected number of handovers as functions of mobility and proxy ratios, as well as the mean correctness of predictions. In detail we treat the more delicate case of these rates in mobile multicast communication. It is obtained that hierarchical proxy environments - foremost in regions of high mobility - can significantly reduce the processing of inter-network changes, reliability of handover predictions is found on average at about $50 \%$.
\end{abstract}

\section{Introduction}

Mobility Support in IPv6 Networks 1 1 has become a proposed standard within these days. Outperforming IPv4, the emerging next generation Internet infrastructure will then be ready for implementation of an elegant, transparent solution for offering mobile services to its users.

At first users may be expected to cautiously take advantage of the new mobility capabilities, i.e. by using Home Addresses while away from home or roaming their desktop 'workspaces' between local subnets. Major scenarios in future IPv6 networks, though, move towards the convergence of IP and 3GPP devices, strengthening the vision of ubiquitous computing and real-time communication. The challenge of supporting voice and videoconferencing (VoIP/VCoIP) over Mobile IP remains, as current roaming procedures are too slow to evolve seamlessly, and multicast mobility waits for a convincing design beyond MIPv6 [2]. 
Synchronous real-time applications s. a. VoIP and VCoIP place restrictive demands on the quality of IP services: Packet loss, delay and delay variation (jitter) in a constant bit rate scenario need careful simultaneous control. Serverless IPv6 voice or videoconferencing applications need to rely on mobility management for nomadic users and applications [3, 4, as well as multicast support on the Internet layer. Strong efforts have been taken to improve handover operations in a mobile Internet, both in the unicast and the multicast case. Hierarchical mobility management [5], 6] and fast handover operations [7], 8] both lead to accelerated and mainly topology independent schemes. In addition to specific performance issues and infrastructural aspects, these concepts cause a different eagerness to operate handovers.

The occurence of handovers is the major source for degradation in mobile network performance and places additional burdens onto the Internet infrastructure. Reducing their frequencies thus promises to ease roaming and to reduce infrastructural costs. In the present work we quantitatively evaluate handover activities with respect to user mobility and geometry conditions.

This paper is organised as follows. In section 2 we briefly introduce the current proposals for improved unicast and multicast mobility. Section 3 is dedicated to our results on handover frequency analysis, derived from analytical models as well as stochastic simulations. Conclusions and an outlook follow in section 4 .

\section{Improved Unicast and Multicast Mobility Management}

\subsection{Hierarchical Mobility and Fast Handovers}

Two propositions for improving roaming procedures of Mobile IPv6 are essentially around: A concept for representing Home Agents in a distributed fashion by proxies has been developed within the Hierarchichal Mobile IPv6 (HMIPv6) [5]. While away from home, the MN registeres with a nearby Mobility Anchor Point (MAP) and passes all its traffic through it. The vision of HMIPv6 presents MAPs as part of the regular routing infrastructure. The MN in the concept of HMIPv6 is equipped with a Regional Care-of Address (RCoA) local to the MAP in addition to its link-local address (LCoA). When corresponding to hosts on other links, the RCoA is used as MN's source address, thereby hiding local movements within a MAP domain. HMIPv6 reduces the number of visible handover instances, but - once a MAP domain change occurs - binding update procedures need to be performed with the original $\mathrm{HA}$ and the $\mathrm{CN}$.

The complementary approach provides handover delay hiding and is introduced in the Fast Handover for MIPv6 scheme (FMIPv6) [7]. FMIPv6 attempts to anticipate layer 3 handovers and to redirect traffic to the new location, the MN is about to move to. FMIPv6 aims at hiding the entire handover delay to communicating end nodes at the price of placing heavy burdens onto layer 2 intelligence. A severe functional risk arises from a conceptual uncertainty: As the exact moment of layer 2 handover generally cannot be foreseen, and even flickering may occur, a traffic anticipating redirect may lead to data damage largely exceeding a regular MIPv6 handover without any optimization. The significance 
of this uncertainty has been recently confirmed by empirical studies [9], where even the use of extensive statistical data under fixed geometry condition led to a prediction accuracy of only $72 \%$.

The two multicast mobility approaches introduced below are built on top of either one of these unicast agent schemes. Minor modifications to HMIPv6 resp. FMIPv6 signaling are requested and both proposals remain neutral with respect to multicast routing protocols in use.

\subsection{M-HMIPv6 - Multicast Mobility in a HMIPv6 Environment}

"Seamless Multicast Handovers in a Hierarchical Mobile IPv6 Environment (MHMIPv6)" 6] extends the Hierarchical MIPv6 architecture to support mobile multicast receivers and sources. Mobility Anchor Points (MAPs) as in HMIPv6 act as proxy Home Agents, controlling group membership for multicast listeners and issuing traffic to the network in place of mobile senders.

All multicast traffic between the Mobile Node and its associated MAP is tunneled through the access network, unless MAP or MN decide to turn to a pure remote subscription mode. Handovers within a MAP domain remain invisible in this micro mobility approach. In case of an inter-MAP handover, the previous anchor point will be triggered by a reactive Binding Update and act as a proxy forwarder. A Home Address Destination Option, bare of Binding Cache verification at the Correspondent Node, has been added to streams from a mobile sender. Consequently transparent source addressing is provided to the socket layer. Bi-casting is used to minimize packet loss, while the MN roams from its previous MAP to a new affiliation. A multicast advertisement flag extends the HMIPv6 signaling.

In cases of rapid movement or crossings of multicast unaware domains, the mobile device remains with its previously associated MAP. Given the role of MAPs as Home Agent proxies, the M-HMIPv6 approach may me viewed as a smooth extension of bi-directional tunneling through the Home Agent supported in basic MIPv6.

\subsection{M-FMIPv6 - Multicast Mobility in a FMIPv6 Environment}

"Fast Multicast Protocol for Mobile IPv6 in the Fast Handover Environments" 8] adds support for mobile multicast receivers to Fast MIPv6. On predicting a handover to a next access router (NAR), the Mobile Node submits its multicast group addresses under subscription with its Fast Binding Update (FBU) to the previous access router (PAR). PAR and NAR thereafter exchange those groups within extended HI/HACK messages. In the ideal case NAR will be enabled to subscribe to all requested groups, even before the MN has disconnected from its previous network. To reduce packet loss during handovers, multicast streams are forwarded by PAR as unicast traffic in the FMIPv6 protocol.

Due to inevitable unreliability in handover predictions - the layer 2 may not (completely) provide prediction information and in general will be unable to foresee the exact moment of handoff - the fast handover protocols depend on fallback strategies. A reactive handover will be performed, if the Mobile Node 
was unable to submit its Fast Binding Update, regular MIPv6 handover will take place, if the Mobile Node did not succeed in Proxy Router inquiries. Hence the mobile multicast listener has to newly subscribe to its multicast sessions, either through a HA tunnel or at its local link. By means of this fallback procedure fast handover protocols must be recognised as discontinuous extensions of the MIPv6 basic operations.

\section{Analysis of Handover Frequencies}

\subsection{Expected Number of Handovers}

As a Mobile Node moves, handovers potentially impose disturbances and put an extra effort onto the routing infrastructure. Thus the expected frequency of network changes can be viewed as a distinctive measure of smoothness for a mobility scheme. The handoff frequency clearly depends on the Mobile Node's motion within cell geometry. Two measures on quantizing mobility have been established in the literature: The cell residence time and the call holding time. Both quantities fluctuate according to the overall scenery and the actual mobility event.

Let us make the common assumption that the cell residence time is exponentially distributed with parameter $\eta$ and that the call holding time is exponentially distributed, as well, but with parameter $\alpha$. Then the probability for the occurrence of a handover from MNs residence cell into some neighboring can be calculated analytically to

$$
\mathcal{P}_{\mathcal{H O}}=\frac{1}{1+\rho}, \text { where } \rho=\frac{\alpha}{\eta} .
$$

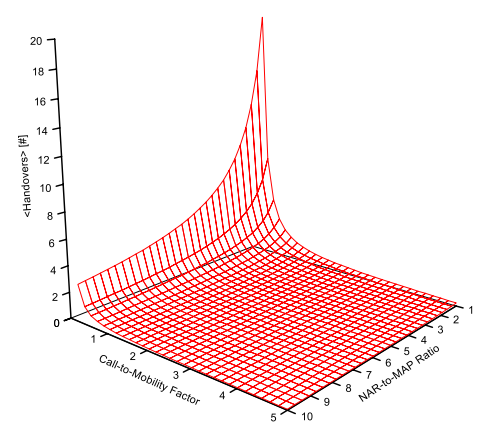

$\rho$ is known as the call-to-mobility

Fig. 1. Expected number of handovers as function of the call-to-mobility factor and the ratio of access routers toMAPs factor [10]. It can be observed that the handoff probability increases as $\rho$ decreases. Note that all probability distributions are homogeneous in space, e.g. $\mathcal{P}_{\mathcal{H O}}$ is independent of the current cell or the number of previously occurred handovers. Spatial scaling can be applied, accordingly.

When comparing Fast MIPv6 and Hierarchical MIPv6 approaches, another distinctive quantity becomes relevant: Whereas FMIPv6 operates handovers at Access Routers, HMIPv6 utilizes MAPs, which form a shared infrastructure. In general one MAP is meant to serve $k$ Access Routers, whence the expected number of (inter-MAP) handovers reduces in a HMIPv6 domain.

Let us assume MAP regions to be of approximately circular geometry. Then the expected cell residence time changes to 


$$
\eta_{M A P}^{-1}=\sqrt{k} \cdot \eta_{A R}^{-1}
$$

and the handoff probability transforms into

$$
\mathcal{P}_{\mathcal{H O}}=\frac{1}{1+\sqrt{k} \cdot \rho},
$$

where $k$ is the ratio of ARs to MAPs.

Now we are ready to calculate the expected number of handovers as a function of the call-to-mobility factor $\rho$ and the AR to MAP ratio $k$

$$
\mathcal{E}_{\mathcal{H O}}=\sum_{i=1}^{\infty} i\left(\frac{1}{1+\sqrt{k} \cdot \rho}\right)^{i}=\frac{1}{k \cdot \rho^{2}}+\frac{1}{\sqrt{k} \cdot \rho} .
$$

It can be observed that in highly mobile regimes $(\rho \ll 1) \mathcal{E}_{\mathcal{H O}}$ is dominantly a function of the inverse of $k$, for low mobility $(\rho \gg 1)$ of the inverse of $\sqrt{k}$ and attains a singularity at $\rho=0$. Fig. 1 visualizes this expectation value for common values of $\rho$ and $k$.

\subsection{Stochastic Simulation of Walking Users}

To evaluate the distribution of handover prediction types, we perform a stochastic simulation of motion within radio cells. The underlying model combines the following ingredients:

Cell geometry is chosen to be of common honeycomb type, i.e. abutting hexagons completely fill the $2 \mathrm{D}$ plane. The ranges of radio transmission are modeled as (minimal) circles enclosing the combs. Thus, regions of prediction are the overlapping circle edges. A geometry of coherent Hot Spots is assumed here, where cells - without loss of generality - are identified with individually routed subnets.

As Walking models a Random Waypoint Model and a Random Direction Model [11] where used, where we consider varying sizes of mobility regions, i.e. squares ranging from cell dimension to infinity. Mobile devices move along (piecewise) straight lines within the preset boundaries, possibly coming to rest at their final destination, even if their current call is ongoing. Note that for finite regions the dynamic of both models is ergodic, whereby our simulated motion is equivalent to a walk continuous in time. Predictions are evaluated along the trajectories, distinguishing their correctness according to the outcome in succeeding steps.

Mean handover occurences for the different geometries are presented in fig. 2 Theoretical results of section 3.1 reproduce nicely for large mobility regions without visible model dependence. Values for small regions are attenuated, as tight geometry borders reduce phase space in 'pushing' trajectories into the inner cells.

Erroneous predicitions as the outcome of traversing prediction regions without actually performing the foreseen handover or handovers initiated from outside of prediction areas are shown in fig. 3. Graph (a) displays in parallel the 


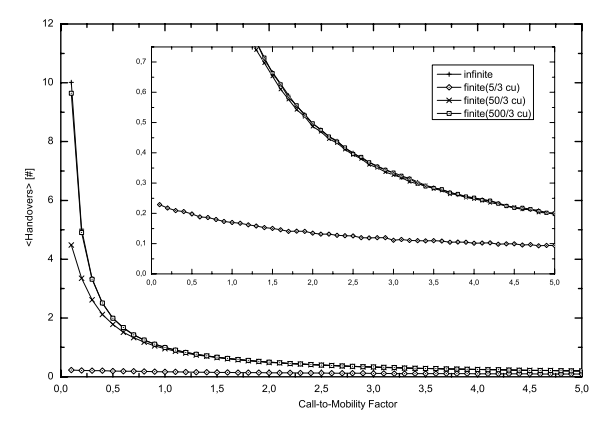

(a)

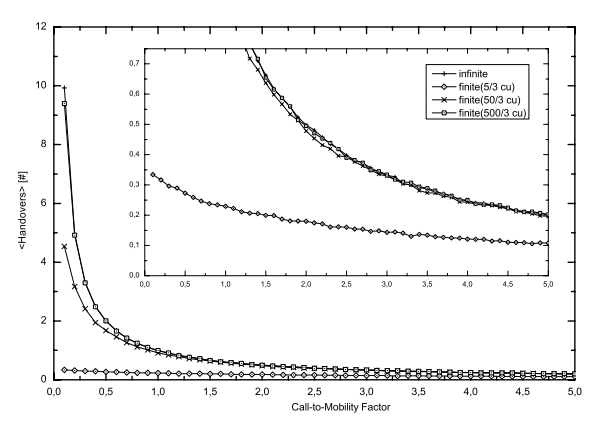

(b)

Fig. 2. Mean frequencies of handovers as a function of the call-to-mobility factor for different mobility regions (in units of celldiameters cu). (a) Random Waypoint and (b) Random Direction Model

percentage of failures from all predictions in the smallest and infinite mobility region for both walking models. From results variing between 10 and $70 \%$ it can be observed that geometry dependence significantly exceeds the influence of the model. Taken as a rough estimate, the average number of erroneous predictions is about equal to the number of correct ones. Thus the reliability of predictive handover schemes does not exceed $50 \%$.

Graph (b) compares corresponding results for the random waypoint model with intermediate mobility region (50/3 celldiameter units) and different radii for the circular radio transmission areas, simulating a variation in access point density. The results for systems with optimal radio coverage, i.e. cell radii equal to transmission ranges, show minimal portions of failure predictions. In general a distinctive geometry dependence becomes visible, as well.

To proceed into a more detailed analysis of the sampled predictions, we differentiated the handover events of a simulated trajectory ensemble (model and parameters as in 3 (b)). Fig. 4 visualizes the mean occurrences of correct predictions, false predictions obtained along the path, as the mobile moves contrarily to forecasts derived from radio signal detection, and erroneous predicitons generated by terminating movement or call at a position, where a handover is expected. The latter yields 'on stop' can be identified as almost mobility independent, resulting in a saturated minimal error rate. Incorrect predictions 'on path' as a function of the call-to-mobility factor in contrast scale in correspondence with the correct indicators. It can be concluded from fig. 3 (a) that their exact values clearly depend on geometric and walking type conditions.

\subsection{Implications}

A common goal in designing HMIPv6 and FMIPv6 has been to approach seamless handovers in mobile IPv6 networks. As was shown in previous work [12], the predictive scheme of FMIPv6 can lead to faster roaming operations, but the 


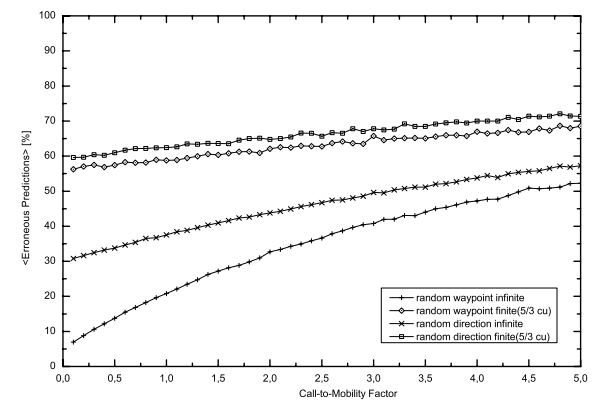

(a)

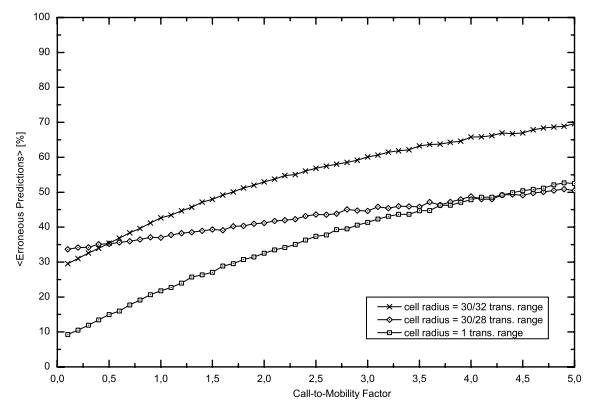

(b)

Fig. 3. Mean relative yield of erroneous predictions as a function of the call-to-mobility factor for (a) different models and geometries and (b) variing transmission ranges

reactive HMIPv6 procedures admits a comparable timing. In scenarios of significant mobility, i.e. $\rho \leq 1$, this advantage may be easily compensated by reducing the number of attained handovers within an HMIPv6 environment up to an order of magnitude. High prediction error rates, as observed from our simulations, place an additional burden onto the infrastructure, since any handover forecast will lead to a negotiation chain between access routers.

This burden notably increases in the case of multicast communication. A preparatory roaming procedure in M-FMIPv6 will initiate a remote multicast subscription, causing multicast routers to erect new branches for the corresponding distribution trees. In combining the results of section 3.1 and 3.2 we face scenarios, where the same (high) mobile movement leads to three handovers in a M-HMIPv6 domain, but about 40 handover processings under the regime of M-FMIPv6.

Another important aspect must be seen in robustness, i.e. the ability of

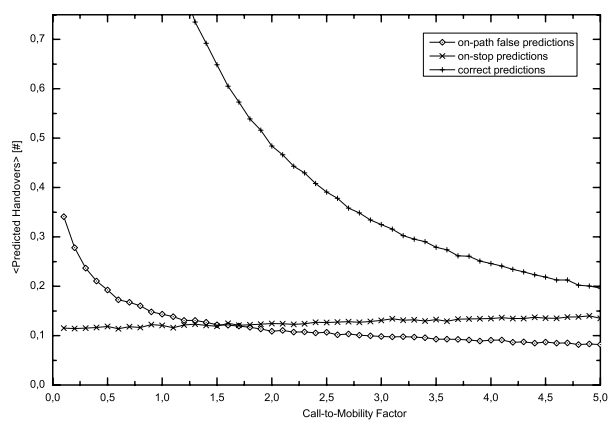

Fig. 4. Detailed view on handovers: Correct predictions, failures along the path and on stop the Mobile Node to cope with rapid movement. In the case of a mobile multicast listener leaving its association before a handover completed, an $\mathrm{M}-\mathrm{HMIPv} 6$ device will remain associated with its previously fully established MAP or Home Agent. On the price of a possible increase of delay, forwarding of multicast data is provided independent of handover frequency. On the contrary M-FMIPv6 forwarding will collapse, as soon as a MN admits a handover frequency incompatible with the signalling completion periods. An M-FMIPv6 device then has to fall back onto MIPv6 by establishing a bi-directional tunnel anew. Meanwhile established services are interrupted. 


\section{Conclusion and Outlook}

In this paper we analyzed common predictive, reactive and proxy mobility schemes w.r.t. their eagerness for handovers. Starting from simple, fundamental assumptions a quantitative study of expected handover occurrences was derived. Prediction reliability was simulated using common mobility models. The 'nervousness' of handovers performed at access routers could be shown to reduce significantly in the presence of Mobility Anchor Points established within the hierarchical MIPv6 approach. This smoothing effect gains additional importance by observing an instability of fast handovers in the case of Mobile Node's rapid movement. The perspective of these results may give rise to further improvements on the smoothing of roaming procedures within the realm of seamlessness, attained at mobile and infrastructure nodes.

\section{References}

1. Johnson, D.B., Perkins, C., Arkko, J.: Mobility Support in IPv6. RFC 3775, IETF (2004)

2. Romdhani, I., Kellil, M., Lach, H.Y., Bouabdallah, A., Bettahar, H.: IP Mobile Multicast: Challenges and Solutions. IEEE Comm. Surv. \& Tutor. 6 (2004) 18-41

3. Schmidt, T.C., Wählisch, M., Cycon, H.L., Palkow, M.: Global serverless videoconferencing over IP. Future Generation Computer Systems 19 (2003) 219-227

4. Cycon, H.L., Palkow, M., Schmidt, T.C., Wählisch, M., Marpe, D.: A fast waveletbased video codec and its application in an IP version 6-ready serverless videoconferencing system. International Journal of Wavelets, Multiresolution and Information Processing 2 (2004) 165-171

5. Soliman, H., Castelluccia, C., Malki, K., Bellier, L.: Hierarchical Mobile IPv6 mobility management (HMIPv6). Internet Draft - work in progress 02, IETF (2004)

6. Schmidt, T.C., Wählisch, M.: Seamless Multicast Handover in a Hierarchical Mobile IPv6 Environment (M-HMIPv6). Internet Draft - work in progress 02, individual (2004)

7. Koodli, R.: Fast Handovers for Mobile IPv6. Internet Draft - work in progress 02, IETF (2004)

8. Suh, K., Kwon, D.H., Suh, Y.J., Park, Y.: Fast Multicast Protocol for Mobile IPv6 in the fast handovers environments. Internet Draft - work in progress (expired) 00, IETF (2004)

9. Song, L., Kotz, D., Jain, R., He, X.: Evaluating location predictors with extensive Wi-Fi mobility data. In: Proceedings of the 23rd Joint Conference of the IEEE Comp. and Comm. Societies (INFOCOM). Volume 2. (2004) 1414-1424

10. Fang, Y., Chlamtac, I.: Analytical Generalized Results for Handoff Probability in Wireless Networks. IEEE Transactions on Communications 50 (2002) 396-399

11. Bettstetter, C.: Mobility Modeling in Wireless Networks: Categorization, Smooth Movement and Border Effects. ACM Mobile Comp. and Comm. Rev. 5 (2001) 55-67

12. Schmidt, T.C., Wählisch, M.: Topologically Robust Handover Performance for Mobile Multicast Flows. In Lorenz, P., ed.: Proceedings of the International Conference on Netwoking (ICN' 2004). Volume 1., Colmar, University of Haute Alsace (2004) 350-355 